\title{
Research planning in the face of change: the human role in reindeer/caribou systems
}

\author{
Gary Kofinas, Gail Osherenko, David Klein \& \\ Bruce Forbes
}

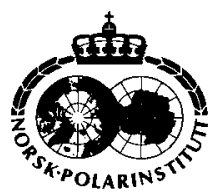

\begin{abstract}
Reindeer/caribou (Rangifer tarandus) constitute a biological resource of vital importance to the physical and cultural survival of Arctic residents since time immemorial. Recent and possible future economic, social and ecological changes raise concern for sustainability of these resources and the well-being of those who depend on them. In February 1999 eighty scientists, reindeer/caribou users and resource managers gathered in Rovaniemi, Finland, for an interdisciplinary workshop to develop a circumpolar research plan that addressed the sustainability of humanreindeer/caribou systems. Small working groups addressed six themes: hunting systems, herding systems, rangeland/habitat protection, minimizing industrial impacts, maintaining the strength of indigenous cultures, and responding to global change. The resulting Research Plan calls for interdisciplinary comparative studies, advancement of tools for assessing cumulative effects, implementation of regional and a circumpolar monitoring and assessment programmes, and cultural studies on the transmission of knowledge. Cross-cutting directives for future research include:

- improving humans' ability to anticipate and respond to change;

- understanding better the dynamics of human-reindeer/caribou systems;

- developing research methods that are both more instructive and less intrusive;

- facilitating open communication among groups with interests in reindeer/caribou resources;

- organizing researchers into a strong, coordinated network;

- re-framing the conventional research paradigm to be more inclusive of differing cultural perspectives.

Three follow-up initiatives are proposed: 1) development of a web-based resource on the human role in reindeer/caribou systems (www.rangifer.net); 2) establishment of a Profile of Herds database to support comparative research; and 3) convening of working groups to address specific problems identified by workshop participants.
\end{abstract}

G. Kofinas \& G. Osherenko, Institute of Arctic Studies, 6214 Fairchild, Dartmouth College, Hanover, NH 03755, USA; G. Kofinas \& D. Klein, Institute of Arctic Biology, University of Alaska Fairbanks, Irving I Bldg., Fairbanks, AK 99775-7000, USA; B. Forbes, Arctic Centre, University of Lapland, Box 122, SF-9610I Rovaniemi, Finland.

\section{Introduction}

From 11 to 14 February 1999, an international group of eighty social and natural scientists concerned with reindeer and caribou (Rangifer tarandus), hunters, herders and resource managers gathered at the Arctic Centre in Rovaniemi, Finland, for an interdisciplinary workshop with a circumpolar scope. The goal of the workshop was to formulate a research plan for future study of the human role in reindeer/caribou systems and to establish a network that links researchers, managers and reindeer and caribou users in research planning.

The Human Role in Reindeer/Caribou Systems Research Plan (sections 4 and 5 of this paper) documents urgent and important issues concerning human-reindeer/caribou systems, pressing questions for future research, and research directives of the Rovaniemi Workshop discussions. This Research Plan also explores implications of the Rovaniemi Workshop for existing human-rein- 
deer/caribou systems research initiatives, as well as how the plan can be sustained as a living document through an interactive, web-based resource. To this end, the Research Plan presented here (and posted at www.rangifer.net) is intended both to generate discussion about the problems associated with collaborative human-reindeer/ caribou systems research and as an invitation for participation in an ongoing circumpolar planning process.

This report is organized in four sections. The first, second and third describe the Rovaniemi Workshop and the process used to facilitate its transactions. The fourth section describes current issues in the human role in reindeer/caribou systems, identifies cross-cutting themes of the workshop, and reports on the six working groups' discussions. The fifth section recommends specific follow-up initiatives for implementing the plan, and addresses how the planning process might be integrated in current and future research initiatives.

\section{About the Rovaniemi Workshop}

\subsection{From international science planning at Dartmouth College in 1995 to the Rovaniemi Workshop on the Human Role in Reindeer/ Caribou Systems, 1999}

The Human Role in Reindeer/Caribou Systems Workshop has its origins at the December 1995 Science Planning Conference of the International Arctic Science Committee (IASC), which was hosted by the Institute of Arctic Studies at Dartmouth College. In the final report of the International Conference on Arctic Research Planning, the working group on the Dynamics of Arctic Populations and Ecosystems made special mention of reindeer/caribou as a keystone species of the North and as the single most important terrestrial resource to Arctic indigenous peoples. The report of that working group went on to recommend a departure from past research initiatives that typically view humans as exogenous to grazing systems, and towards the consideration of humans as a central component. More specifically, the working group identified four broad research questions worthy of future interdisciplinary investigation. These questions (Table 1) served as the starting point for the research planning process undertaken in Rovaniemi.
Table l. Key questions for Arctic researchers, as formulated by the Dynamics of Arctic Populations and Ecosystems Working Group of the International Conference on Arctic Research and Planning.

1) When there is a change within ecosystems in the quality or quantity of resources available to human users, how do humans respond?

2) When humans alter the way they interact with the environment, how does the environment respond? Can we predict the impact of this feedback on the various components of the ecosystem?

3) Can we predict the impact of external influences (ranging from pressures and constraints applied by outside authorities to long-range transport of pollutants into the human-reindeer/ caribou food chain) on northern resources and people?

4) How do changing institutions, rights, rules and regulations regarding access to, management of and ownership of land, water and other natural resources affect the health of populations and ecosystems?

\subsection{Toward an inclusive, interactive model of research planning}

"Science planning" is sometimes perceived by the public and practised by agencies, academics and governments as an exercise of elite (and occasionally isolated) scholars who produce a single document guiding research funding initiatives for a three- to five-year period. Following the International Arctic Science Planning Conference, an international, multi-disciplinary group of researchers and reindeer/caribou users took up IASC's charge to address the question of future human-reindeer/caribou systems research, but did so with a different orientation. From the beginning, those involved sought to redefine science planning as a process to be undertaken through direct interaction with reindeer and caribou users, and with those who have special expertise in the range of relevant disciplines. The goal of science planning from this approach is to create an ongoing process of networking people and their research-related resources, to identify and address common problems, and to orient investigations through common analytical frameworks. Fundamentally, this approach to science planning aims to increase the responsiveness of research activities to changing environments and their socioeconomic conditions, and to enhance opportunities for circumpolar exchange of information and collaboration.

This approach to science planning is not intended to be a straightjacket that might dictate or limit future research opportunities. It does, 
however, prioritize the concerns of researchers and reindeer/caribou users to funding agencies. As is clear from past experience, successful funding proposals addressing international circumpolar issues are best supported with collectively formulated research priorities. Thus, the intended audience for this research plan is broad. We anticipate that project funders will use it to identify current trends in the field of human-reindeer/ caribou studies, resource user communities will use it to learn about the methods employed by other groups to address problems similar to their own, and professional researchers will consult it to formulate agendas, find research partners and cooperate with relevant user groups.

\section{The workshop format and the participants}

The Human Role in Reindeer/Caribou Systems Workshop was launched by an international and interdisciplinary steering committee (Table 2). Funding for the workshop was provided through grants from several sources (Table 3), primarily from the US National Science Foundation, the John D. and Catherine T. MacArthur Foundation, the Trust for Mutual Understanding, and the International Arctic Science Committee.

The goals of the workshop were threefold:

- To produce a science plan that identifies gaps in prior research and existing cooperative arrangements, and proposes a strategy to fill the gaps;

Table 2. The Human Role in Reindeer/Caribou Systems Workshop Steering Committee

Gail Osherenko, Dartmouth College, USA (Workshop Coleader)

David Klein, Univ. of Alaska Fairbanks, USA (Workshop Co-leader)

Hugh Beach, Univ. of Uppsala, Sweden

Vladimir Etylin, World Reindeer Herders Association, Russia

Nicholas Flanders, Dartmouth College, USA

Gail Fondahl, Univ. of Northern British Columbia, Canada

Bruce Forbes, Univ. of Lapland, Finland

Gary Kofinas, Dartmouth College and Univ. of Alaska Fairbanks, USA

Margarita Magomedova, Institute of Plant and Animal Ecology, Russia

Elna Sara, World Reindeer Herders Association, Norway

Pete Schaeffer, Kotzebue Tribal Council, USA

Peter Usher, Wildlife Management Advisory Council (NWT), Canada

Kofinas et al. 2000: Polar Research I9(I), 3-21
Table 3. Sponsors of the Human Role in Reindeer/Caribou Systems Workshop

The United States National Science Foundation

The John D. and Catherine T. MacArthur Foundation

The International Arctic Science Committee

The Trust for Mutual Understanding

The Arctic and Alpine Terrestrial Ecosystems Research Initiative (ARTERI), a European Community Concerted Action group

The Walter and Duncan Gordon Foundation

Canada's Department of Indian Affairs and Northern

Development (DIAND)

The Alaska Conservation Foundation

- To publish a symposium volume in a peerreviewed journal on the topic of the human role in Rangifer systems;

- To build a network that links scientists, user communities, and those managing and protecting reindeer/caribou systems.

Our objective was to engage individuals who collectively represent a cross-section of different Arctic geographic areas, indigenous user groups, academic researchers and those who could speak to the range of conditions and their researchrelated questions associated with the study of the human role in reindeer/caribou systems. Given the limitation of time and money and the goal of working towards an interactive process, participation was restricted to about 80 individuals.

Workshop participants came from the Nordic countries (32\%), North America (30\%), Russia $(26 \%)$, and elsewhere (5\%). The approximately eighty people total participating included researchers $(59 \%)$, reindeer herders and leaders from reindeer herding organizations $(11 \%)$, caribou hunters and leaders of Native groups (9\%), professional resource managers $(9 \%)$ and representatives from non-government organizations $(3 \%)$. Of the researchers, nearly two-thirds were natural scientists $(62 \%)$, with the remainder $(38 \%)$ representing the various social sciences. Indigenous users included leaders from several North American First Nations, the chairpersons of Canada's two most long-standing caribou comanagement boards, the President of the Finnish Saami Parliament, the President of the Association of World Reindeer Herders, the President of the Union of Russian Reindeer Herders, as well as owners or directors of state and private herds. (See www.dartmouth.edu/ arctic/rangifer/people/index.html for a full list of workshop participants.) 


\subsection{The workshop process}

To facilitate indigenous-science and trans-disciplinary interactions, workshop plenary presentations were limited, with the bulk of the workshop's schedule allocated to working group sessions. Capturing key themes of the study of humanreindeer/caribou systems, the workshop was organized around six foci:

- hunting systems

- herding systems

- rangeland and habitat protection

- minimizing industrial impacts

- keeping indigenous cultures strong

- responding to global change

(See www.dartmouth.edu/ arctic/conf/ for a listing of the plenary sessions and poster and oral presentation abstracts.)

An important design feature of the workshop was the inclusion of a forum in which Arctic residents shared their perspectives on the problems and concerns facing resource users, and identified perceived needs for future studies. Users of local resources are often keenly aware of conditions that are relevant to the advancement of knowledge and the research needed to inform public policy processes. Arctic peoples' involvement in the workshop also reflected an appreciation that resource users are most likely to be affected by the findings and implications of research. At the Human Role in Reindeer/Caribou Systems Workshop, special attention was devoted to concerns and needs of indigenous reindeer and caribou users whose cultural identity and economic welfare depend on the sustainability of reindeer/caribou resources.

The Rovaniemi Workshop concluded with reports from each of the six working groups. Following the workshop, eight workshop participants reviewed and coded working group transactions to identify cross-cutting themes and directives of the Rovaniemi research planning process. These are presented in the sections that follow, along with a summary of Native plenary panelists' comments, noting their implications for research planning.

\section{The Research Plan}

\subsection{A long and intimate relationship}

Reindeer and caribou grazing systems have long been exploited for food and other subsistence commodities. The relationship between reindeer/ caribou and people extends back to prehistoric times through an ecological process that is among the most complex of any system in the biosphere. Many of the diverse indigenous cultures spanning the Arctic and sub-Arctic have co-evolved with reindeer and caribou, which provide food, shelter and transportation. These peoples include (but are not limited to): in Eurasia, Saami, Nenets, Komi, Khanti, Dolgan, Nganasan, Yukagir, Even, Evenk, Sakha (Yakut), Chukchi, Koryak and Chuvan; and in North America, Gwich' in, Dene, Metis, Cree, Chipewyan, Innu, Naskapi, Yupiit, Iñupiat, Inuvialuit, Dogrib, Koyakan and Inuit.

Today, Arctic residents face a combination of dramatic changes to the biological resources that are crucial to their physical and cultural survival. Indigenous societies of the circumpolar region define themselves largely in relation to their use of particular living resources (e.g. caribou, reindeer, marine mammals) that historically have been and remain critical to their physical, economic and spiritual well-being. Among these, caribou and reindeer feature prominently in the oral traditions of hunting and herding peoples, and continue to serve as links to their past traditions and future ambitions.

\subsection{Urgent and important issues}

Potential and current changes to human-reindeer/ caribou systems are today multi-dimensional. Large-scale non-renewable resource development projects are occurring or planned in many regions of the Arctic and sub-Arctic. Long-range transport of pollutants from outside of the region is considered to be causing habitat changes and has generated significant fear among those who are regular consumers of reindeer and caribou. Climate change, which is likely to be greatest in polar regions, raises questions regarding its effects on the growth and distribution of forage plants, the distribution and movements of animals, its implications for insects which affect caribou and reindeer energy reserves, and users' access to and movement with animals at important times of the annual cycle.

Fundamental changes in political and legal structures have also been dramatic, and are affecting indigenous Arctic residents who depend on reindeer/caribou resources. In the Russian North, the demise of many state farms and the rise of the market economy and privatization are 
changing the roles that indigenous peoples must play and the rules by which they must operate. The former state supported economic and supply system of distribution has collapsed, creating a need for new methods of marketing, distribution and herder support. In some cases these problems are coupled with the unscrupulous decisions of government officials whose interests are at odds with reindeer herding peoples. In Canada, creation of new political entities like Nunavut and implementation of numerous land claim settlements have altered relations between local indigenous communities and the state, and at the same time transformed communities that are a part of these arrangements.

It has been argued that increasing human needs for stability in food availability, clothing and transportation in the Eurasian North put pressure on wild reindeer in Eurasia and, in combination with changes in climate, brought about the shift from dependence on wild reindeer hunting to reindeer husbandry. Whereas the transition to reindeer herding added stability and sustainability to the antecedent hunting societies, conversion from a subsistence economy to commercial reindeer husbandry in this century added new and difficult challenges to modern resource management of these northern grazing systems.

The current status of reindeer herds across the Russian North is by no means uniform. On the Yamal Peninsula, the population of semi-domesticated reindeer has risen steadily in the postSoviet period, resulting in serious questions regarding the capacity of remaining rangelands to support the herds. In contrast, semi-domesticated herds on the Chukchi Peninsula have dwindled while wild reindeer herds have increased, making it extremely difficult for herders to prevent their stocks from joining wild herds.

For the northern peoples of Canada and Alaska who depend on hunting of caribou, a major issue is how' the influence of the western industrialized world has changed their relationship to the resource. North American hunters have always experienced shifts in the size and distribution of caribou herds. Prior to this century, the use of a suite of resources, trade networks and geographically broad social relationships eased the effects of fluctuations. Now commercial opportunities, wage employment and government welfare also buffer changes in resource availability.

Indigenous rights and local involvement in resource management are important issues tied to caribou and reindeer grazing systems. Increased access to these systems by southern populations, enclave developments, and culturally inappropriate management policies have spurred northern peoples to seek clarification of their rights and to reassert their role in management of both wild and domesticated herds. The results of these efforts are reflected in passage of new laws, recent court decisions, land claims agreements and the establishment of co-management regimes. The longterm effects of these changes on the sustainability of caribou and reindeer grazing systems are largely unknown.

Reindeer herding and caribou hunting societies developed a body of knowledge about these northern grazing systems and adapted to the presence and absence of animals through opportunistic, mobile hunting strategies. Arctic industrial development in this century led to the expansion of the Arctic human population, establishment of permanent settlements away from the coast, adoption of new technologies and the addition of wage work. Many a new settlement and industry across Siberia depended on reindeer herders for meat and, originally, for transport as well. The Soviet authorities thus "industrialized" reindeer herding to facilitate the development of the Soviet North. Now, privatization and the shift to a market economy in Russia pose new challenges for indigenous herders. These changes have added new complexity and pressures to the relationship of humans to Arctic ecosystems. Overall, these changes have made the Arctic grazing system more dependent upon the global economy. Modern science has greatly expanded understanding of the relationships among Arctic species. There has, however, been little integration of knowledge about trophic levels with ecosystem and landscape level studies, nor an appreciation of the value of local and traditional knowledge in understanding these systems. Increased pressure for exploitation of ungulate populations and pressure for changes in patterns of reindeer husbandry in the North have been generated in recent decades without development of an understanding of the complexity of human-reindeer/ caribou systems sufficient to enable planning and management for their sustainability.

\subsection{Local and regional perspectives and their implications for research planning}

Perspectives of indigenous user panelists at the 
Rovaniemi Workshop provide a more intimate account of important and urgent problems of human-reindeer/caribou systems, and offer insight on how forces for changes manifest themselves in the daily lives of hunters and herders. The concerns expressed by Native workshop participants also reflect how conditions differ by region, while concurrently reflecting problems associated with initiating a circumpolar research planning effort.

Among the most striking and desperate concerns expressed in Rovaniemi were those from Russia. These participants spoke of the abject poverty in which many herders currently subsist, the disarray at the local level which has followed from the collapse of former state-controlled management systems, the absence of recognized rights for indigenous herding people, and the increased industrial activity which has resulted from current open-access conditions. Following from this situation have been dramatic declines in habitat forage quality and emergent pressures on local herders to abandon traditional herding ways of life for more urban-based lifestyles. Anthropologists working in the region framed these conditions in more blunt terms - as a "crisis." Alexey Polezhaev, an ecologist from the Institute of Biological Problems of the North, put the conditions in more political terms of "local control" by speaking to the need for "political and social measures to regain the social and economic life of reindeer husbandry." These desperate concerns, described both by state and private reindeer users of Russia, raise the question of how people incurring such conditions can concern themselves with a circumpolar research planning process when their basic survival needs are in question. Clearly, making the link between herders' immediate social, economic and political needs and research is critical.

Fenno-Scandian Saami participants, on the other hand, highlighted problems in their region of decreasing viability of reindeer herding and multiple forms of encroachment by non-reindeer users on important pasturelands. Described in pragmatic terms, this situation is related to acquiring adequate financial resources to "survive" in modern society, while facing the reality that reindeer husbandry is maintained at low levels of productivity. From the indigenous perspective, the problem of low productivity is the result of herders being forced into smaller pasturelands, resulting in heavier grazing pressures due to encroachment of development on grazing areas and bi-national policies on fencing and transborder herd movements. Johan Matis Turi, President of the World Reindeer Herders Association, spoke particularly of the efforts of Saami peoples to organize and become a viable political force, and the need to view Native herders as part of the heritage of Arctic natural systems. In spite of considerable efforts in this area, securing adequate rights from central governments to sustain herding remains elusive.

Native users of Alaska spoke of a different set of concerns. Vera Morris of Kiana, a small and remote village in north-western Alaska, described past conditions in which declines in caribou populations were perceived by biologists and managers as being primarily the result of Native over-hunting and waste. Native Alaskans today live with imposed state government hunting regulations, and limited formal rights when working -with agencies in managing caribou and restricting urban-based sport hunters who presently have political dominance over the Alaskan state legislature. As a result, most local families must modify the traditional rules by which Native Alaskans relate to wildlife. As Morris put it,

My son is fifteen and his dad is teaching him to hunt. Jim [his dad] learned from his father where to find caribou, about gun and boat safety, about butchering animals. Now, as we're teaching our son, there's a lot more to it. We have to talk with him about sports hunters, boundaries and regulations, about diseased animals and protecting rights for the future.

Echoing similar issues, Davie James, Director of the Natural Resources Department of the Council of Athabascan Tribal Governments in north-western Alaska, spoke of his organization's nascent efforts to increase community research capacity, and how this goal is being undertaken through acquisition of grants in spite of limited government agency support. Concerns driving community-controlled research agendas in his region include environmental health, long-range transport of contaminants, low-flying military and civilian aircraft, and the loss of elders along with their rich knowledge of land and caribou.

For Native Alaskan workshop participants, these political and environmental conditions have direct implications to the research planning process initiated in Rovaniemi. Following from the protracted political conflicts of Alaska, today 
there is limited trust among researchers, managers and indigenous resource users and, therefore, great suspicion about collaborative projects involving non-locals.

Canadian caribou hunters who have considerable experience working through a myriad of newly established land claims settlements indicated a set of research related concerns from a slightly different perspective. Joe Tetlichi, Chairman of the Porcupine Caribou Management Board, noted that while some communities have undertaken their own documentation of traditional knowledge, it remains uncertain whether such projects will carry weight with territorial and federal agencies when enacting management policy. Violet Camsell-Blondin, a leader of the Dogrib First Nation in Northwest Territories, expressed concerns regarding development projects that are typically reviewed in impact assessment processes individually, and not adequately assessed at a landscape level. As she stated, "We are concerned about the cumulative effects of all activities - mines, hydro, roads, outfitters, and fishing lodges ... We have to be sure we do not sacrifice our environment, as caribou is our food."

Canadian First Nations people pointed out that language is fundamental to knowledge, and raised the problem of finding common constructs in research, as well as when framing the Rovaniemi Workshop's objectives. Allice Legat, an anthropologist who works on traditional knowledge projects with the Dogrib First Nation of NWT, spoke to the limitations of translating indigenous concepts into western languages and the problems of "mining" indigenous knowledge systems as data sources without regard for their underlying cultural perspectives.

Canadian participants went on to point out that the term "science plan," as used by the workshop organizers, is perceived as exclusionary to many indigenous hunters, and limits participation of those who identify their system of knowledge as distinct from the scientific methods of western inquiry. Anthropologist Igor Krupnik added that there are also hazards in generalizing a monolithic notion of "western science," and not recognizing the diversity of traditions of learning among and within the many academic disciplines. In response, Rovaniemi Workshop participants agreed to dispense with the use of the term "science plan" and in the future refer to the group's engagement in a "research planning process."

\subsection{Cross-cutting themes of workshop transactions}

Cross-cutting themes of the Research Plan represent broad categories of concern that emerged in workshop discussions. In many cases, these themes do not provide the detail necessary for actual research planning of specific study projects. They do, however, serve as guiding principles by which research should be formulated, implemented and evaluated (Table 4). As noted, these themes were identified through a coding exercise undertaken by a subgroup of participants after the close of the workshop. It is recommended that these themes be cross-referenced with the individual working group reports.

Table 4. Guiding questions for future research.

- How does the research improve the ability to anticipate and respond to change?

- Will the research add to our understanding of how the system functions?

- To what extent does the study advance methodological practices?

- Does the research provide resources that facilitate better communication among key players?

- Does the research help to organize people into a stronger and better coordinated network?

- Does the research re-frame the problem in a way that speaks to current needs and revises outdated paradigms?

4.4.1. Improve the ability to anticipate and respond to change: Today's unprecedented socio-economic, environmental, and political changes in the Arctic and the role of humans in reindeer and caribou systems underscore the need to investigate more thoroughly and articulate more clearly the loss, risks and benefits associated with specific policy choices. Today's changes are both novel and multi-dimensional, and thus require research initiatives to examine social and economic responses to the physical and biological landscapes, as well as to new economic structures. There is a need to advance knowledge of the indicators of change in order to predict critical levels of impact and the thresholds for cumulative effects. These research efforts should not, however, overestimate our existing knowledge base. While much knowledge has been gathered about the relationship between humans and reindeer/ caribou, there remains great uncertainty in many important areas. As pointed out in Rovaniemi, many management boards with access to rela- 
tively good data struggle to answer their most basic questions (e.g. Should hunters harvest a decreasing population? When should managers attempt to manipulate a population? Do radio collars affect caribou behaviour?).

\subsubsection{Understand better the functionality of} human-reindeer/caribou systems: Understanding systems' functionality is the most discussed theme of research among professional researchers, and a key focus of inquiry for determining how best to respond to change. Working groups stressed this theme, though each elaborated different aspects of the system. Those focusing on minimizing the impacts of industrial development spoke of the need to identify pathways of contaminants and the functional response of organisms at lower trophic levels. The rangelands and habitats working group, however, discussed the relative importance of complexity with respect to cumulative effects on critical habitat, while social scientists and resource managers highlighted the need to examine the effectiveness of different institutional arrangements, to reduce or at least coordinate jurisdictional complexity, and to build social capital. In order to understand how human-reindeer/caribou systems function, the particular focus of each field of research must be developed and each field of research must be cognizant of the others.

To advance understanding of human-reindeer/ caribou systems there is today a dire need to build more and better databases of information sources (i.e. meta-databases) that provide for more indepth analyses. Future data collection efforts are recommended through the development and maintenance of regional and circumpolar monitoring tools. For monitoring of human-Rangifer systems to be useful, data collection protocols need to be established and integrated with other research programmes. Two models for exchanging data and making regional comparisons of particular note for Arctic researchers are the Arctic Monitoring and Assessment Programme (AMAP, see www.grida.no/amap) and the International Tundra Experiment (ITEX, see www.systbot.gu.se/research/itex/ itex.html). The use of remote sensing offers another area of research that has considerable potential for contributing to the development of circumpolar monitoring tools (e.g. satellite imagery and the use of normalized difference vegetative index [NDVI]). There has been some initial advancement in the integration of resource users' knowledge into ecosystem monitoring systems, but more methodological development is needed. These elements of a monitoring programme offer much promise, particularly if used in tandem with geographic information systems (GIS) mapping, and to calibrate, validate and refine simulation models through both retrospective analysis and special case studies.

Utilizing comparative methods in circumpolar studies of human-reindeer/caribou systems offers special opportunities, both with single discipline and interdisciplinary research endeavours. A comparative approach could potentially contribute to our understanding of system functionality by contrasting wild and domesticated reindeer herds, disturbed and undisturbed landscapes, and different institutional environments such as different community-state power sharing management regimes. The comparative method also offers the advantage of lending itself to quantitative and qualitative analysis, as well as hybrid approaches that are interdisciplinary in nature and incorporate combinations of the two.

Comparative analysis also offers rich opportunities to address important theoretical problems. For example, circumpolar comparisons raise the question of the extent to which findings can be extrapolated across populations, regions and regimes, and the extent to which investigators can scale local findings up to landscape and regional levels. From a somewhat more applied perspective, there are potential benefits from testing the assumptions, constructs, methods and findings of sensitive habitat identification, both between wild and domestic populations and between local knowledge and research science.

Finally, the study of the human role in reindeer/ caribou systems should take more seriously the role of culture as a determinant of system behaviour and human response to change. While reindeer and caribou research has typically focused on "natural systems" and viewed human communities as exogenous in its analysis, formal and informal social institutions (i.e. rules in use), levels of trust between parties, the flow of information, and groups' access to financial resources and political power affect ecosystem dynamics and resource conservation practices as well. Bridging the gulf between these behavioural elements of human society with the biophysically-oriented dimensions of a grazing system remains one of the greatest challenges to future researchers. 
4.4.3. Develop new methods for doing research: Participants of the Rovaniemi Workshop repeatedly raised the point that while the substance of research findings is critical, so are the methods used in achieving them. Central to understanding complexity and functionality of human-reindeer/ caribou systems is the use of methods that are holistic in approach. Today, while holism is commonly espoused as a goal for research, most projects fall far short of being interdisciplinary; few models of research are readily available for achieving interdisciplinary objectives. Mentioned as an example of working towards these objectives was the Sustainability of Arctic Communities Project, funded by the US National Science Foundation and involving 22 researchers of different disciplines and four Native caribou hunting communities of Alaska and Canada. This integrated assessment has drawn on research science and local knowledge to produce simulation models for the purpose of improving the ability of scientists and locals to understand and discuss how forces of change may affect communities meeting their sustainability goals (see www.taiga.net/sustain).

Comparative circumpolar research also requires advancements in methodological approaches for comparing case studies, so treatment of data sets collected with differing methods may be compared and analysed. New sources of data derived from remote instruments (e.g. satellite imagery) offer considerable promise, although improved methods are needed to ground-truth findings, understand the relationships with Rangifer population-level response, and account for regional and local variability.

Methodological advancement towards the coproduction of knowledge by indigenous communities and researchers was also a key theme explored in Rovaniemi. Indigenous people and researchers alike called for methodological development that results in future studies that are both rigorous and useful, as well as less intrusive. Issues of power and power sharing are important in this enterprise, both with respect to allocation of research resources (e.g. human expertise, access to funds), as well as the terms with which local knowledge is perceived to be legitimate. The debate on this topic revealed two camps. One group proposed a co-production process in which specific cultural perspectives on change would be exchanged and mutually included in common projects. Others were less sanguine about colla- borative projects, stating a preference for the segregation and distancing of local and sciencebased research initiatives, with exchanges confined to discursive interactions.

Three substantive research questions illustrate the issues surrounding intrusiveness of research, the use of local people's knowledge and the achievement of holistic studies:

- Is it possible to assess a reindeer/caribou population without counting the herd and, if yes, how?

- What incentives foster good interdisciplinary inquiry among scientists?

- How might user communities best be involved in research so as to respect locals' traditions, ensure rigour and relate findings to a public policy process?

On another front, there is a need for advancement in methods for documenting subsistence uses of herds and hunters, especially those that identify the territories and resources used by indigenous peoples and that provide data essential to protect their rights to land and resources.

4.4.4. Facilitate better communication among key players: Conventionally, researchers view communication as outside the domain of their research activities, and necessary only among key players in the "peer review" of publishable articles. This approach has proven problematic in the areas of reindeer and caribou studies, especially as related to risk assessment, but also in the achievement of effective shared decision-making.

In Rovaniemi, researchers, managers and resource users alike called for an increased emphasis on information exchanges that make research activities and their findings more accessible to lay people, and enable residents of the Arctic and those beyond to understand, contribute to and use research findings. While the World Wide Web is an emergent vehicle for achieving better information exchange, people at the margin are typically without such services and are culturally oriented to face-to-face interactions. Regardless of the medium of exchange, it is clear that information transfers are important, both in allowing resource users access to the findings of the research community and in creating meaningful dialogue between groups that promote mutual understanding, respect and new discoveries.

Assuming that communication is best when it is two-way, there is a need to foster research projects 
that bring greater prominence to the voice of resource users and legitimacy to their perspectives on ecological, social and economic systems. Participants of the Herding Systems Working Group suggested a set of projects, entitled "Let the People Speak", to provide resource users their own stage for voicing their perspectives and presenting historical data in their own way. Others, seeking to blur the distinction between the subjects of research and the investigators, called for a set of "Natives Study Natives" projects, using video ethnography that supports region-to-region circumpolar learning and cooperation. Most important, these ideas speak to the fundamental principle that those directing research, whether they are indigenous or not, should include hunters and herders in all phases of research, and look to them when defining normative measures (e.g. defining community well-being and sustainability).

Such efforts should not be confined to indigenous peoples studying other indigenous peoples' approaches to problem-solving. The scientific community has much to gain from exchanges of ideas about research methods, and fora promoting such exchanges are needed. As noted by those in Rovaniemi, this is particularly evident for professional scientists of Russia where financial resources and access to higher technologies are limited.

4.4.5. Organizing into a stronger and better coordinated network: The workshop in Rovaniemi was the first gathering of scientists, reindeer and caribou users, and mangers to address research in human-reindeer/caribou systems in an interdisciplinary forum. Today, the interest in the human role in reindeer/caribou systems is widely dispersed, although there is increasing focus on achieving greater collaboration. The benefits of a stronger and more coordinated network of those interested in the study of human-reindeer/caribou systems include new opportunities for circumpolar comparisons, exchanges of research methodologies and the identification of common patterns of change and proven strategies for coping with them. Moreover, this coordination can be used in acquiring greater recognition of funding needs. Clearly, such efforts depend on the creation the exchange of information via an effective network.

4.4.6. Re-framing the issues and revising the paradigm: Fundamentally, the Rovaniemi hu-
man-Rangifer Research Plan calls for a rethinking of conventional approaches to planning and carrying out research in a way that embraces the complexity of Arctic systems, their rapid state of change, and the increasing threats to humanreindeer/caribou relationships. As indicated in the cross-cutting themes outlined above and the individual reports of working groups, below, this shift in thinking requires that underlying assumptions of many conventional research efforts be questioned and re-framed. In many cases, future research should reflect better the chaos that is part of all natural systems, and accept disequilibrium as a natural dynamic of Arctic systems. Shifts in paradigm in the policy sciences are also in order, requiring a re-framing of political analysis which has historically looked at human needs to one that accounts for rights and duties of social institutions, organizations and social networks, and their emergent patterns of behaviour. Yet in many respects, a tremendous body of knowledge is currently available, and serves as the basis for expanding our horizons to meet the present-day challenges of people and reindeer/caribou. The challenge requires more an adaptation to new conditions, rather than an abandonment of all that has been accomplished before.

\subsection{Working group reports}

Six topic areas were the subject of working groups in Rovaniemi. They are: hunting systems, herding systems, minimizing industrial impacts, habitat and rangeland protection, protection indigenous cultures, and responding to global change. Each group grappled with its respective problem area to identify key issues and important questions, and to generate research recommendations. No specific format was provided for each group to follow, so the reports from each group differ. In the sections that follow, report summaries are presented in point form for brevity and readability.

\subsubsection{Hunting Systems Working Group report: As} technological advances make their way in society, cultural traditions and hunting systems change. The challenges these changes create are related to working out alternative strategies that ensure future survival of cultural systems, rather than a one-dimensional preservation of old ones. With this general theme in mind, the Hunting Systems Working Group identified five major problem areas, and within each noted research questions, 
comments, arguments and possible research priorities. For the most part, these discussions focused on barren-ground Rangifer, although it was acknowledged that considerable hunting of caribou and wild reindeer occurs at more southern latitudes. The members of the Hunting Working Group expressed discomfort with the lack of circumpolar representation in their group, as only two Europeans were present and no Russians attended the session. Thus, important issues relating to hunting as occurring in these regions are likely to have been overlooked. Below is a list of some of the key points of the working group, organized by topics.

\section{Hunting concerns regarding the transformation of hunting systems}

- The conflicts and increasing competitions between sport (i.e. urban-based recreational hunters) and indigenous subsistence hunters raise questions regarding the extent to which they can co-exist and how future conflicts will be resolved. In some areas of Alaska, the conflicts are resulting in acts of violence.

- To understand the current conditions and overall dynamics of hunting systems, there is a need to document more completely hunters' participation in hunting economies. Specifically, there is a need to acquire better measures of the time individuals, families and households devote to hunting, the geographic areas travelled by individuals, the effect of jobs on these activities, the conditions in which collective hunts are implemented, and the extent to which collective hunts meet the needs of communities and households.

- There is a need to identify changing hunting patterns and relate these changes to regions and cultural orientation.

- Changing societal values at large are critical for anticipating the future security of hunting peoples' way of life. Changing societal values raise questions about how caribou and wild reindeer hunting can best cope with anti-hunting groups, the work of extreme "animal rights" organizations, and gun-control legislation; and how to shape public opinion to ensure that hunting rights are protected.

\section{Biological issues related to caribou management in hunting systems}

- Answers to some of the most basic caribou management questions are lacking. When should a group manipulate a population? What is the best method for estimating annual mortality? Is it possible to implement and then measure a hunting strategy that corrects for natural morality? Is it possible to monitor abundance without counting animals and, if so, how?

- Industrial activities, eco-tourism and sport hunting activities suggest a need to understand better one of the most mysterious aspects of caribou ecology - the environmental forces affecting annual and seasonal herd movements and distribution.

- Crippling rates of hunted caribou have been based historically on the subjective assessment of managers and biologists and used in projecting changes in herd population. Methods for establishing crippling rates need to be evaluated.

- Management of caribou for hunting requires a closer assessment of the fundamental concepts. For example ecological variability (e.g. variability in snow cover) is not well understood in caribou management.

- Many hunting people state that caribou and muskoxen "do not get along." In some regions expanding muskoxen numbers are negatively correlated with caribou numbers. Do caribou and muskoxen compete for forage or do they avoid each other?

\section{Hunting and research methods}

- Hunting peoples' traditional values regarding caribou use call for respectful treatment of animals. Some hunters feel that there is a need to develop less intrusive methods for assessing population parameters.

- There is a need to develop new ways for social and natural scientists to interact in the study of game management. Both have the potential of complementing each other.

- The inclusion of "traditional ecological knowledge" (TEK) in caribou studies and wildlife management is commonly recommended, but the methods for achieving this end are not well developed.

\section{Subsistence economies}

- Changing economies in Russia raise the question of the possible blends between hunting and herding reindeer and caribou.

- What are the social and ecological problems related to transition from wild to domestic management systems?

- What are the most important drivers of change in the transformation of a subsistence economy?

Policy studies: institutions and jurisdictional complexity

- Several regions face problems related to inconsistent jurisdictional policies applied to fugitive Rangifer resources. What are the design principles in resource regimes that account for successful (or unsuccessful) jurisdictional coordination?

- When are formal rules and regulations necessary, and in what areas and in what conditions is it best for the system to function with informal institutions? From a slightly different frame, what is the effectiveness (and appropriateness) of basing management on tribal laws vs. state laws vs. federal laws when applied to indigenous hunting?

- How do social institutions affect hunting traditions and hunting patterns? What is the circumpolar experience in this area?

- What are the traditional and contemporary philoso- 
phies and management strategies of hunting societies in game management (e.g. predator control), and bow might they contribute to a public policy process?

- What is the relationship between the context in which management occurs and the appropriateness of a formal board-oriented (i.e. co-management) arrangement? What is the relationship between contextual features of management and success in the different functions of management?

\subsubsection{Herding Systems Working Group report:} Herding systems are part of the larger whole that comprises northern systems, and reflect changing physical, socio-economic and institutional environments that are affected by individual and group aspirations. Cognizant of these conditions and with greater interaction with other non-local populations, herders increasingly express the need to "know." Knowledge of these conditions has utility to herders in coping with change. Holistic approaches to knowledge acquisition are of special value to herders. The Herding Systems Working Group drew on these overarching concerns to identify research and methodological issues related specifically to herding systems.

\section{Research themes}

- The high diversity of reindeer herder peoples and their respective systems of pastoralism represent an area of strength for herders, but also uncertainly. There currently exist no central information source that serves as a central database on herding people. Workshop participants identified the need for a circumpolar herding "census."

- Documentation cases (examples) of herding societies throughout the circumpolar North are needed.

- Documentation of projects should reduce the role of non-herding people in telling the story of herding people, and to the greatest extent possible should draw on the words of herders themselves. "Let the Herders Speak" is the recommended title of this set of projects.

- Comparative studies that blur the distinction between research subjects and researchers should be associated objectives.

\section{Methodological issues}

- There is a great deal that can be done with existing knowledge, and without the collection of new primary data.

- The research processes of making new discoveries, continuing with and drawing from current initiatives, collating existing data and disseminating available findings are necessary and should not be overwhelmed with efforts to launch new research programmes.

- The framing of past research questions has been conducted with limited direct consultation with local herders research; formal and information systems are needed to establish closer links between these groups so the framing of questions are informed by herding peoples.

- Beyond the problem of framing specific research questions are underlying theoretical concepts of science that are at times culturally foreign and in some cases inappropriate to herders who are the subject of research. Research endeavours should be sensitive to these differences and, where possible, incorporate the categories and terminology of herders.

- Formal organizations like the World Reindeer Herders Association, as well as more local herder organizations, should assume a formal liaison role in this process.

\section{Herding/hunting conflicts}

- With increased range pressure and greater interaction among reindeer herders and wild caribou hunters, there is a need to identify mitigative measures for reducing conflicts. This is particularly true in North America where the interaction of greatly expanding wild popula'tions and herders' stocks has resulted in economic losses to herders.

\subsubsection{Rangeland and habitat protection working} group report: The quality of grazing rangelands and the potential degradation of habitat conditions represent an aspect of human-reindeer/caribou systems research that has historically been underappreciated. In the modern-day context, this topic emerges as the single most important and controversial area of conflict. Conditions which lead to change in range conditions can result from a density-dependent response to increases in herd population (as has been described for rangelands in Russia and the George River Herd in eastern Canada), but also the result of shifting human land use patterns (e.g. hydrocarbon development and roads). Concurrent with these can be shifting climatic forces. In some cases, the cumulative effects of these forces add to the system's complexity, thus rendering current methods of analysis ineffective. Participants of the Rangeland and Habitat Protection Working Group grappled with these issues to address five key topic areas. Specific project recommendations were also identified to address attendant problems. Issue areas identified are understanding habitat stability, creating monitoring systems of habitats, identification of critical habitats, understanding spatial integrity and understanding animal-habitat interactions.

\section{Rangeland habitat stability and projects}

- While on a local level there is a need to focus on 
human effects on ecosystem processes and their relationship to habitat productivity, at the regional level there is a need to understand better cumulative effects and their expression as local impacts. Scaling to the global level also needs to be understood better, considering climatic and pollution related effects and feedback.

- These three levels of analysis - local, regional and global - translate into three recommended research projects that would: 1) determine the scalability of sitelevel results to landscapes and regional levels; 2) determine the implications of landscape-level changes to Rangifer productivity, while addressing the implications of change to management; and 3) determine the importance of habitat complexity for cumulative effects.

\section{Monitoring systems}

- There is a need for regional and circumpolar habitat monitoring tools that are improved with calibration, validation, refinement of models, retrospective analyses and special case studies.

- Monitoring systems for human-reindeer/caribou systems need to be integrated with other existing programmes to ensure adequate transfer of information and knowledge. Projects like ITEX and AMAP offer good examples and opportunities for rich interdisciplinary exchange.

- Remote sensing and local knowledge represent two areas in which monitoring tools can be advanced.

- A specific need is to develop a large-scale monitoring tool for lichen-dominated communities.

\section{Identification of critical, sensitive and important habitats}

- Formal and standard criteria are needed for this identification process. Elements should include animal use and value by humans, ecological performance and habitat productivity. Critical habitat identification needs to be applicable at the herd level and integrated with classic scientific and local knowledge systems.

- Advancing the state of knowledge in this area can be achieved through a comparison of critical habitat identification concepts, methods and results (not limited to Rangifer, nor the Arctic), a comparison of local and scientific knowledge systems for wild Rangifer, and a comparison of key habitat selection for domestic Rangifer populations.

\section{Problems of spatial integrity}

- Understanding the value of spatial integrity in the performance and health of grazing systems is especially important in light of current trends towards increased habitat fragmentation through economic development.

- The research needs in this area have special significance in addressing corridors between critical habitat, in maintaining those connections, and in identifying mitigative policy options in the face of proposed habitat modifications.
- Associated with such research is the need to view caribou populations as super-organisms, and to understand carefully the thresholds at which cumulative effects are apparent.

- A recommended approach in addressing these research problems is to model the effects of mitigation options on critical habitat connectivity, including ecological, economic, sociological and political phenomena.

\section{Animal-habitat interactions}

- Identification of potential pathways is needed to assess better human risk and functionality of grazing systems. Nitrogen cycling, disease and their relationships to human health and herd productivity are examples. Projects that compare undisturbed and disturbed landscapes would serve to identify detectable feedback.

- Comparisons of wild and domestic herds may provide insight into the relative strength of feedback mechanisms in different systems and help to identify the strengths and differences among these systems.

- Qualitative assessment tools are needed to understand habitat state effects on animal performance. Integration of local knowledge and science to assess these should be a central feature of this inquiry.

\subsubsection{Minimizing the Impacts of Industrial} Development Working Group report: Industrial development within the ranges of the reindeer and caribou populations is expanding at a dramatic rate, raising questions about their ultimate impacts and how development activities can be modified to minimize negative effects on grazing systems. Participants of this working group completed a cursory comparison of development activities within different regions (e.g. large-scale gas development in the Yamal, the Prudhoe Bay and Kuparuk oil fields, smaller scale oil development in the National Petroleum Reserve of Alaska, expansive diamond and other mineral mines in the Bathurst, the Bluenose and Beverly Herd ranges, and the extension of road, tourism and power line corridors in Norway.) These discussions highlighted current fragmentation of knowledge and opportunities for comparative studies.

\section{Cumulative effects and existing development}

- There is a need to summarize and synthesize understanding of cumulative effects from existing developments in order to project what may be expected from future proposed developments of a similar nature (i.e. oil/gas fields, open pit mines, etc.) Such studies would best be undertaken where there is a reasonably long history of development under presumed restrictions to minimize environmental impacts, such as the Prudhoe 
Bay Oil complex and Quebec hydroelectric development.

- Studies should try to determine what criteria are valuable in assessing disturbance and cumulative effects.

- Specific investigations would monitor ongoing development to categorize and chronicle the cumulative effects as they occur, from pre-initiation through termination and rehabilitation. While long-term funding commitments from responsible agencies are necessary to accomplish this, it is recognized that obtaining this type of project sponsorship is difficult.

- Comparative research of existing development projects and their impacts of grazing systems are highly recommended. Three types of comparative studies are proposed: a) similar developments in different countries (e.g. diamond mining in Northwest Territories Canada and Russia); b) different types of development (e.g. oil production and mining); and c) industrial development in markedly different environments.

- Cumulative effects are recognized as an area of study that is central to the study of existing development activities, and which requires greater clarification botk with respect to conceptual development and formulation of research methodology.

Behavioural responses of caribou/reindeer to industrial activities

- Behavioral studies are needed to identify the responses of caribou/reindeer to a variety of development activities and structures under a variety of environmental conditions. Studies of this type have been and are being done (e.g. Prudhoe Bay, Dempster Highway, hydro development in Norway), and should be continued and broadened to include situations not yet covered, including seasonal differences.

- Studies should address questions regarding the degree of habituation possible in caribou and reindeer to specific disturbances (e.g. roads, traffic, low-flying aircraft, pipelines, hikers and skiers in calving areas, etc.) in different terrain, vegetation (forested vs. tundra), seasonal conditions (presence or absence of snow), and the presence or absence of predators, including humans.

Societal responses to effects and integrated assessment of industrial development

- Studies should explore the effectiveness and deficiencies of legally mandated impact assessments through the testing of "models" as predictive tools.

- Comprehensive interdisciplinary studies of the full impacts of development on a particular geographic/ political region are needed. These studies should be undertaken as retrospective analyses to delineate differences and similarities of impacts. These studies offer special promise, especially if they include adequate detail.

\section{Case studies of impact assessments}

- Research is needed to analyse present forms of impact assessment with the objective of highlighting their pitfalls and deficiencies, and moving towards more adaptive approaches.

\section{Research capacity analysis}

- Methodological problems exist where professionals in particular regions are not able to identify, analyse and communicate regional problems. This is particularly problematic in Russia, where a wealth of data is available but access to sophisticated analytical tools is limited. Studies in these regions should best be undertaken by teams of researchers focusing on a regional basis, but ideally completed on a country-wide basis, with one or more of the team coming from outside the region and sharing experience with development assessment under more controlled conditions.

\section{Protected areas}

- Research is recommended to focus on the principal components of establishing protected areas or a protected areas network (where "protected area" means a geographically identified region that is managed according to a set of sensitivity criteria). Components of these studies could include: a) documentation of caribou/reindeer seasonal movements and use of ranges and a review of possible changes associated with climate change; b) means of assigning "values" in an ecological, cultural and economic terms, with psycho-social studies investigating risk perception and transformation of values, for people residing in both rural and urban areas; c) documentation of existing institutional approaches toward caribou/reindeer habitat protection; and d) analysis of the means for recognizing and exploiting decision-making authorities/regimes.

- The product of protected habitat studies should be the identification of critical habitat units that can provide the basis for guiding and/or constraining development to avoid habitat degradation. Such studies should also provide the background necessary for demonstrating the relative importance of critical habitat to grazing systems - including the people dependent upon them - to policy makers.

\section{Comparison of "useful" information}

- Studies are needed to evaluate the relative utility of various types of information needed in impact assessment and habitat protection. A central aspect of this area of research is to identify the minimum information needed in advance of authorization of a development project.

\section{Institutional management effectiveness}

- Research is needed to explore a range of existing institutional arrangements with respect to their appropriateness and effectiveness in managing humancaribou/reindeer systems in the face of development. Studies should assess the appropriate role of user communities, industry, government, NGOs and non- 
locals in the application, exploration, extraction and restoration phases of development.

\section{Pollution and contaminants}

- The working group defined pollution as "matter that arrives from near or far which is absorbed by the biota to contaminate the environment." With regard to longrange pollutants, there is a need for studies and monitoring that identify the origins and levels of longrange pollutants that affect caribou. Examination of specific pollutants, their pathways and their longevity in the system (e.g. the half-life of radio nuclides) should be a part of this process.

- Vegetation studies are especially important for understanding the effect of pollution on forage, particularly lichen. Related to this area of inquiry are studies on lichen physiology to understand how they respond to pollutants. Knowledge of the effects of pollution on Arctic and alpine vegetation was characterized as low, with lichen physiology described by workshop participants as a black box when compared to the knowledge of vascular plants. Background level studies should determine existing or native levels of common contaminants. For example, cadmium levels may be the result of geo-chemical anomalies of specific sites.

- Caribou/reindeer ecology and pollution. Specific studies are need to relate range ecology (e.g. seasonal movements/forage) to types of pollutants and how they affect animals. These studies would include duration of exposure to site-specific pollutants, considering range use patterns and harvesting activities, seasonal variation, and exposure due to change in diets. Leaching of pollutants into the environment is also important.

- Studies are deemed necessary to understand the effects of nitrogen pollution on plants, ozone and changes in geo-chemical cycles, especially as nitrogen pollution relates to increased sensitivity of plants to abiotic stresses. It was noted that nitrogen pollution may go hand in hand with climate warming, increasing growth of some plants through fertilization and warmer or longer growth season that may be detrimental to other plants which thrive under low nutrient availability and cool conditions (e.g. lichens and sedges - both important seasonal forages for reindeer and caribou).

- Major changes in plant communities may result with birch shrub of low forage quality increasing to the detriment of high quality forage species. Increasing ultraviolet-B (UV-B) due to thinning of the atmospheric ozone layer at high latitudes will affect plants differentially. Those that cannot adapt (e.g. leaf thickening to protect active photosynthesizing tissues) may die out and those that can adapt may be of lower forage quality. Studies have been initiated in this area in northern Sweden, but more effort should be directed.

- There is a need to identify key indicators of pollution levels that are important to grazing systems and human uses of reindeer and caribou.

- Studies are required that examine the intake, trans- formation and bio-accumulation of pollutants/contaminants in reindeer/caribou. These are important because not all pollutants are potential contaminants and some that may have no significant effect on plants or reindeer/ caribou may move through the food chain to humans, where they may become contaminants.

\section{Human health and contaminants}

- Existing information on human health risks from contaminants are insubstantial and inappropriate for the Arctic. Medical studies relevant to the Arctic and its people are required regarding health risks from exposure to contaminants. Existing and new medical research centres in the North need to focus on this important issue.

- Human contamination should be monitored and the sources and pathways identified.

- Studies should determine appropriate and effective means of communicating health risks of contaminants to northern people. There is a specific need for sociologists and psychologists with experience working in northern communities to undertake these studies.

\section{Lichen studies}

- Lichen were recognized as a key species in grazing systems, yet little is known about the ecology of these plants.

- There is a need to investigate the dynamics of the structure and function of lichen-dominated communities. (Cross reference with Habitat Protection and Range Use Working Group.)

\section{Disturbance recovery}

- Studies would follow recoveries of post-disturbance systems to illustrate potentiality for recovery following particular disturbance events. Studies could address ecological, sociological, economic, cultural and political aspects of post-disturbance response and recovery

\subsubsection{Indigenous Cultures Working Group report:} Cultural studies on the human role in reindeer/ caribou systems, unlike single disciplinary approaches, offer a unique opportunity for holism. Workshop interest in this topic area was substantial, with the Indigenous Cultures Working Group proving to be the most well attended and the most diversely composed, with respect to both geographical as well as disciplinary representation. Epistemological questions regarding knowledge and cultural systems were at the heart of many of the discussions, as it was recognized that indigenous peoples continue to redefine their identity in modern-day contexts by asserting the uniqueness of their perspective, and advancing their political stature by contributing knowledge in resource management and other public policy 
processes. Knowledge was expressed by members of this working group as a process of learning, including the transfer of knowledge through time and between individuals. Loss of knowledge was identified as fundamental to culture change, with its maintenance linked to the protection of reindeer/caribou societies. The extensive transactions of this work group were distilled into a set of four pressing areas of research:

1) What are the mechanisms and processes by which knowledge is transmitted between generations and adapted to changing conditions in reindeer/caribou societies? This question addresses social stability, continuity and flexibility, and questions the concepts of traditional and modern. In particular, attention needs to be paid to rapidly changing gender roles.

2) What is the range of cognitive, philosophical, moral and practical presuppositions embedded with the diverse ways of knowing that come to bear on reindeer/caribou societies?

3) How is different knowledge used in arriving at decisions affecting reindeer and caribou people? This question includes the study of political, legal and economic systems of dominant societies as well as indigenous societies. Comparative studies are recommended as the preferred method of analysis.

4) Comparative studies are needed in the construction of knowledge and worldview of reindeer/caribou people as they concern variability in the reindeer/caribou populations and behaviour. This includes understanding knowledge as a process, the role of cosmology and symbolism, and the distinction between knowledge as information and knowledge as wisdom.

\subsubsection{Responding to Global Change Working} Group report: The Global Change Working Group focused on a broad set of topics that allowed comparison of differing perceptions of change in different regions. While North American researchers stated their keen interest in and concern about climate as a driver of change, Europeans and Russian researchers argued that overgrazing and habitat degradation are more critical forces. Participants agreed that anthropological (i.e. globalization of economies, changing social structures, transformation of political power) and institutional issues (formal and informal policies, rules in use, conventions, norms influencing behaviour) were significant in all

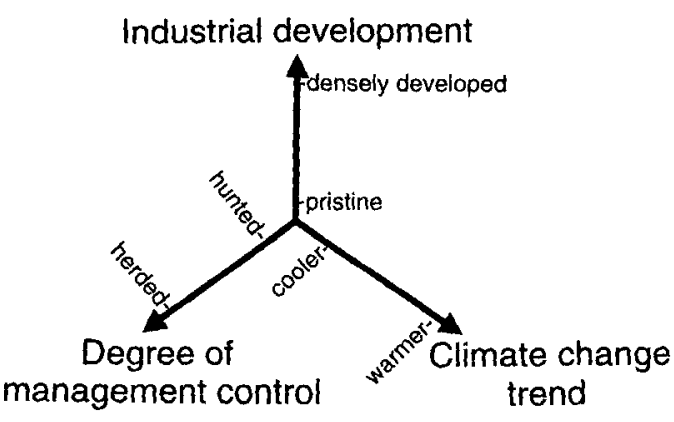

Fig. 1. Three-dimensional framework for the comparative study of global change in human-Rangifer systems.

cases. In an attempt to capture differing forces for change in different regions for analysis in circumpolar research, the group developed a three-dimensional framework (Fig. 1). Several key issues and questions were articulated:

- A meta-database (i.e. source of information sources) is needed that provides the basis for regional comparisons of change, as well as methodological techniques that will modify available data to make them comparable.

- Institutional dimensions were identified as an underrepresented element of most global change studies.

\section{Beyond Rovaniemi: outstanding questions and follow-up initiatives}

How should the energy generated at the Human Role in Reindeer/Caribou Workshop and the ideas articulated in this Research Plan be best directed? Clearly, the ideas expressed in this document have no future if ways are not identified to incorporate them into existing research initiatives and implement them in some form as a coordinated circumpolar project. Current financial limitations as well as political barriers make establishment of a full-blown international office for study of the human role in reindeer/caribou systems research unlikely in the near future. Perhaps more appropriate is a call to build informal links among interested parties, and explore new ways to share resources, exchange ideas and launch collaborative studies.

To date, several major research initiatives that focus on reindeer and caribou systems are striving to realize these goals. (See www.dartmouth.edu/ $\sim$ arctic/rangifer/resresources/programs.html for a 
listing of research projects.) These initiatives are expanding our knowledge of:

i) the ecosystem relationships of caribou and reindeer in specific areas of the Arctic;

ii) the human connections to these Arctic reindeer/caribou systems, including their cultural, social and economic importance;

iii) the effectiveness of different systems of management of the caribou and reindeer populations and their forage resources; and

iv) the political and legal issues facing land use in the Arctic and their importance to reindeer/caribou systems.

The Rovaniemi Workshop has already generated several important research proposals, including a comparative analysis of North American caribou herds and hunters, an initiative by CAFF to include a Rangifer systems component in its biodiversity monitoring programme, a circumpolar UNEP programme including the assessment of the impacts of infrastructure on grazing systems, an ethnographic video exchange between Kola herders of Russia and Gwich'in hunters of Canada, and a large participatory European study of reindeer herding systems involving herders and scientists.

Whereas these research initiatives represent a significant start in fulfilling essential research needs, they are not comprehensive in their coverage of the Arctic, nor in filling the critical information gaps that exist. At present there is no circumpolar assessment of rates of industrial encroachment on sensitive Rangifer habitat, no overview assessment of encroachment impact on hunting and herding systems, and no single source of information on the status of North American barren-ground Rangifer populations and their hunting communities. While extensive data on reindeer grazing in Russia exist, no single source provides high-resolution maps of pasturelands. Such maps are essential for industrial interests and foreign investors as well as those researchers and resource users preparing social and environmental impact assessments of proposed development. Additionally, there is currently no Arctic-wide monitoring and assessment programme to provide an indication of the environmental health of reindeer and caribou as a subsistence and commercial resource.

To begin meeting the objectives outlined in this research plan, three initial follow-up activities are proposed: 1) establishment of a web-based re- source on the human role in reindeer/caribou systems; 2) the creation of a "Profile of Herds" initiative for use in future comparative studies; and 3) convening of working groups to address specific topic areas identified by workshop participants as worthy of special attention.

\subsection{An interactive, web-based human-reindeer/ caribou systems resource}

A clear directive from those participating in the Human Role in Reindeer/Caribou Systems Workshop was to maintain the newly established network and research planning process initiated in Finland. To this end, the Institute of Arctic Studies at Dartmouth College has established a web-based resource located at www.rangifer.net (Fig. 2). The website's components include:

- "Research plan" - a posting of this research plan with a feedback function, allowing readers to comment on its parts and access the comments posted by others.

- "Join the discussion" - invites readers to join an e-mail discussion forum, as well as the Network of People in Human-Rangifer Systems.

- "Events and news" - a posting of current events and news, e.g. information about conferences.

- "Rangifer resources" - annotated links with selected web-based resources such as those connected with current and past Rangifer research, human uses of reindeer and caribou, caribou comanagement arrangements, agency research programmes, reindeer herding organizations and educational resources.

- "Profile of Herds" - intended to provide an overview of circumpolar human-reindeer/caribou systems, including population estimates of animals, sensitive habitats, management regime, groups of resource users, government jurisdictions, historic record of harvest levels, regionsspecific conflicts etc.

- "People in Rangifer systems" - listing of individuals involved in research, including indigenous leaders, managers, academics and others.

At the time of this printing, the website represents more of an opportunity than a fully developed resource. Its success depends upon the contributions of members of our community and the availability of resources to maintain the effort. The website's utility remains limited to those with access to computers and the Internet; thus a high priority for development aid must be the enhance- 

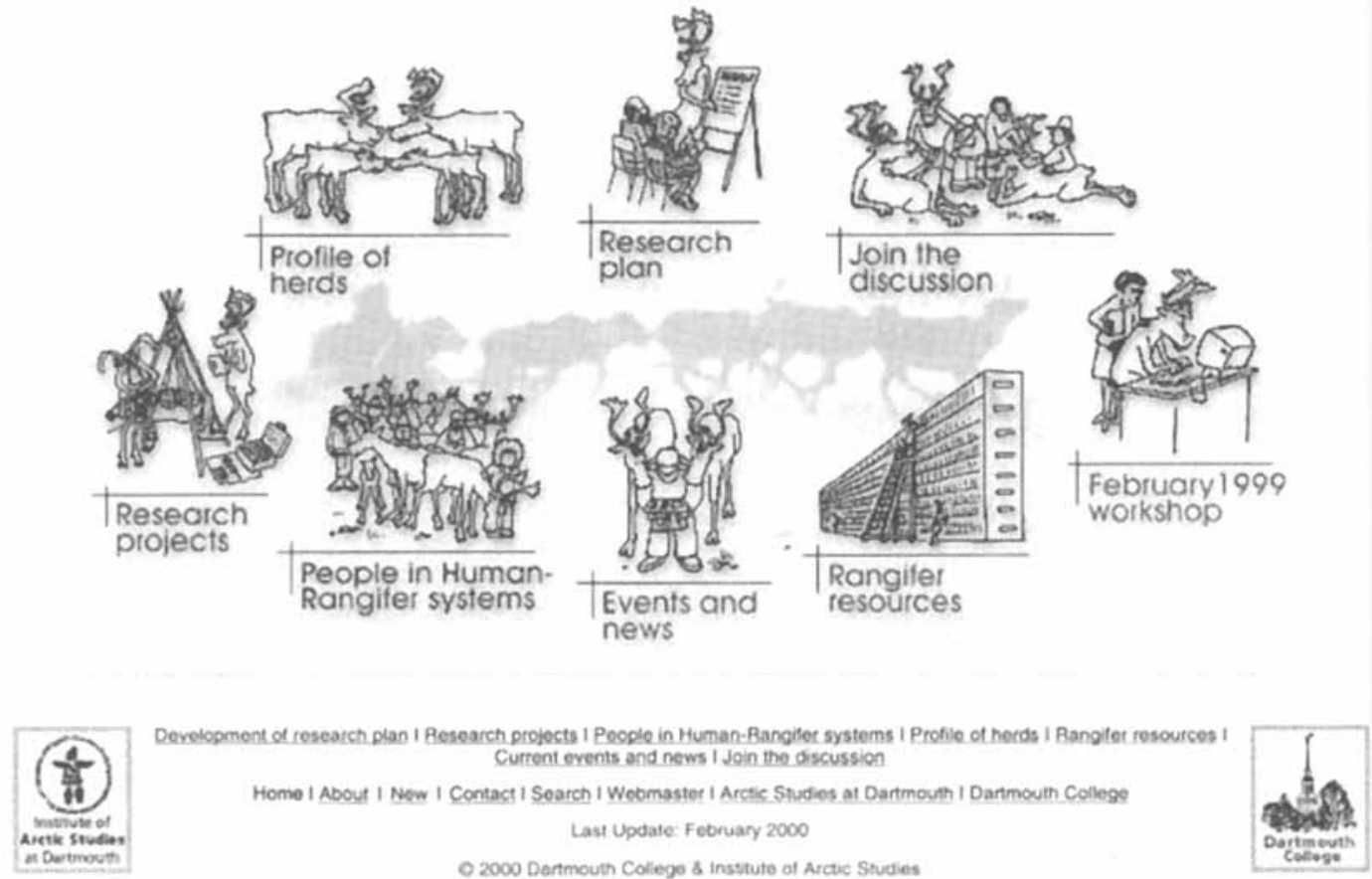

Fig. 2. The home-page of www.rangifer.net, the Internet site established by the Institute of Arctic Studies at Dartmouth College.

ment of telecommunication infrastructure. Until such time outlying communities have such access, materials will also be distributed in printed form.

\subsection{The Profile of Herds initiative}

Compilation of a database and a meta-database related to human-reindeer/caribou systems represents a key first step in undertaking future circumpolar comparative analyses. The database and meta-database should include (but not be limited to):

- historical population trends and current status

- productivity

- status of important habitat

- key areas of management uncertainty

- reported use levels

- user groups

- herding and hunting culture groups and uses of reindeer/caribou

- government agencies with management jurisdiction
- management regime characteristics

- relevant legislation

- past, current and potential future industrial development

- past and current management conflicts

- current regional research priorities

- active researchers and their organizational affiliations

- key regional contacts

At present, the www.rangifer.net website provides a placeholder for this information. We propose that key resource people from different circumpolar regions collaborate to compile the database, and that information be made accessible to researchers, students and the general public. This compilation will be undertaken in close cooperation with CAFF's biodiversity monitoring initiative. We envision that as new innovations to the World Wide Web emerge (e.g. better use of audio and video, new analysis tools, faster connections), there will be considerable opportunities to develop and broaden this resource. 


\subsection{Circumpolar working groups on special topics}

We also propose that several small working groups, each addressing specific topics identified in Rovaniemi, be convened to facilitate future circumpolar discussions and research planning. Based on the transactions in Rovaniemi, several topic areas are worthy of consideration. Their foci include:

- evaluation of methods for assessing cumulative effects of industrial activities on reindeer/caribou and hunting and herding societies;

- the transition of knowledge among and between reindeer and caribou user culture groups;

- transformation of herding and hunting economies;

- principles of effective institutional design in sustaining human-reindeer/caribou relations;

- nested and linked programmes for monitoring and assessment of human-reindeer/caribou systems.

This list of topics serves as a starting point for further discussions. In presenting it, we acknowledge that resources from more affluent countries are needed to support the participation of the less affluent, and that such reallocation and sharing are the only way in which a stronger circumpolar network can be achieved.

\section{Conclusion}

The Human Role in Reindeer/Caribou Systems Workshop was an historic event in its focus and mix of participants. While its Research Plan sets an important research agenda, it also illustrates a new model in circumpolar research planning. Innovative and important, the workshop and the Plan call for the rethinking of conventional research concerning human-caribou/reindeer relationships and improving efforts to resolve urgent and important problems. The success of this enterprise ultimately hinges on:

- finding resources - building and empowering research teams, locating the requisite funding to finance and disseminate their work;

- taking risks - being less territorial, more collaborative, less parochial and more interdisciplinary;

- showing respect - learning to learn collectively, sharing the outcomes of collective learning, and recognizing the differing contributions of all.

Acknowledgements. - Although the authors assumed primary responsibility for drafting the Research Plan, it was the participants of the Human Role in Reindeer/Caribou Systems Workshop who provided its content 


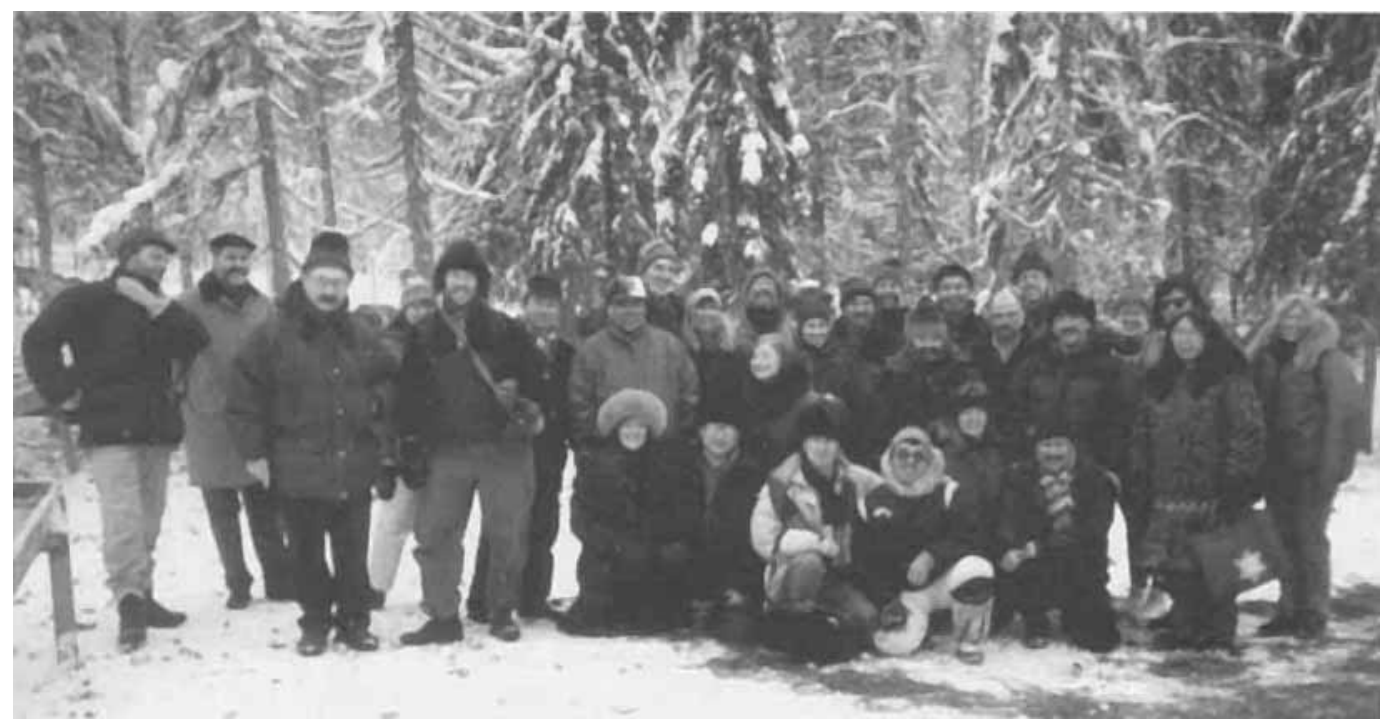

Several participants taking a break from the workshop during a trip to a reindeer farm not far from Rovaniemi. Standing (left to right): Peter Aastrup, Manfred Boelter, Leonid Baskin, Susan Lee, Bruce Forbes, Leonid Kolpashchikov, David James, David Klein, Heather McIntyre, Gary Kofinas, Valentina Baskin, Deborah Robinson, Brad Griffith, Jack Kruse, Don Russell, Joe Tetlichi, Doug Urquhart, Brian Johnston, Mike Ferguson, Allice Legat, Denise Geoffroy, Vera Morris, Randy Myers; kneeling: Gail Fondahl, Nikolay Karpov, Anatoliy Alexeev, David Kritterdlik, Micheline Manseau, Yuri Chernoev. Photo: G. Kofinas.

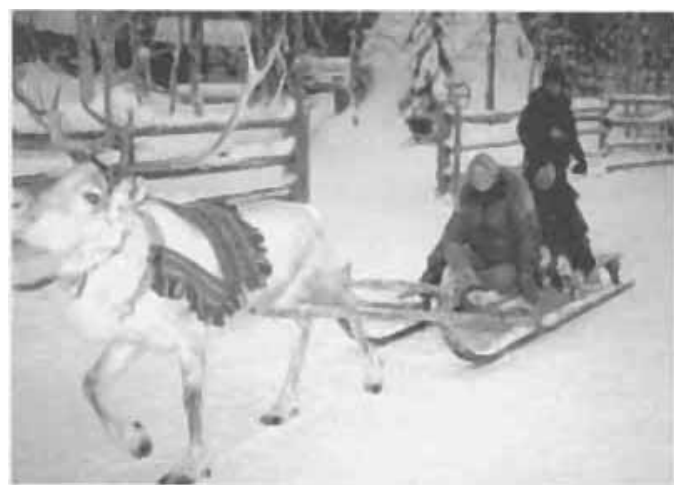

Randy Meyers and Vera Morris of western Alaska take a sledge ride during the field trip to the reindeer farm. Photo: G. Kofinas.

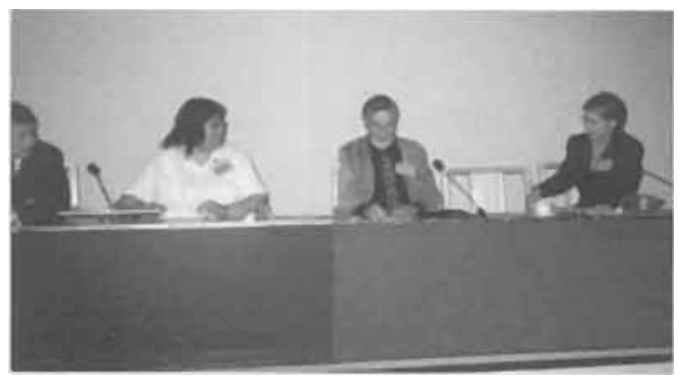

Dmitrii Khorolia, Violet Camsell-Blondin, David Klein and Allice Legat discuss indigenous priorities for research following the keynote presentations. Photo: G. Kofinas.

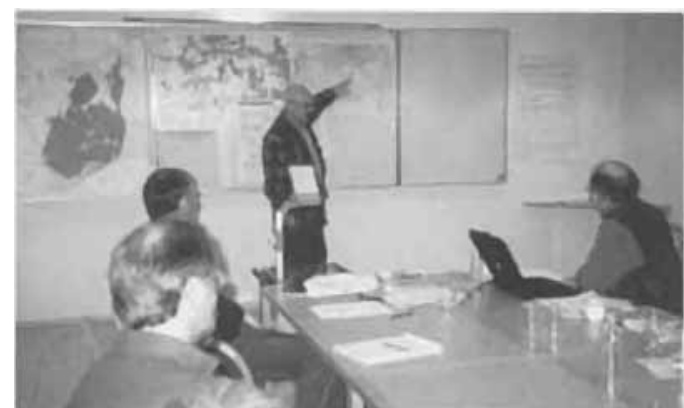

Doug Urquhart takes a break from writing notes while Leonid Baskin points out a region of concern (north-west Siberia) during the working group session on industrial development. Photo: G. Kofinas.

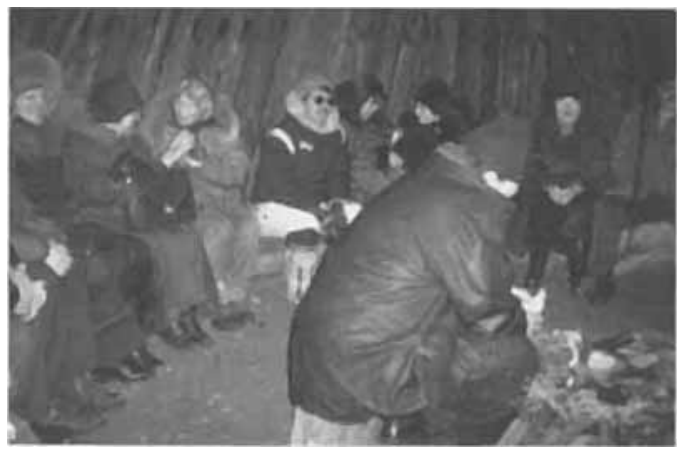

Some workshop participants warm up around a fire in a Saami kota (wooden teepee) during the field excursion to Nivankylä. Photo: G. Kofinas. 